\title{
The Applicability of Intraoperative Neuromonitoring in Patients with Preoperative Motor Weakness during Spine Surgery
}

\author{
Jae Meen Lee ${ }^{1}$, Dong Hwan Kim², Hwan Soo Kim², Byung Kwan Choi ${ }^{2}$, In Ho Han ${ }^{2}$ \\ ${ }^{I}$ Department of Neurosurgery, Seoul National University Hospital, Seoul National University College of Medicine, Seoul, \\ ${ }^{2}$ Medical Research Institute, Pusan National University Hospital, Pusan National University College of Medicine, Busan, Korea
}

Objective: The purpose of our study is to evaluate the success rate and feasibility of intraoperative neuromonitoring (IONM) focusing on transcranial motor evoked potential (TcMEP) monitoring for patients with preoperative motor weakness in spine surgery. Methods: Between November 2011 and December 2013, TcMEP and somatosensory evoked potential (SSEP) monitoring were attempted in 130 consecutive patients undergoing spine surgeries for cervical or thoracic cord lesions. Patients ranged in age from 14 to 81 years (mean \pm standard deviation, $56.7 \pm 14.8$ years), and 84 patients were male. The success rates of both SSEP and MEPs monitoring were assessed according to the preoperative Medical Research Council (MRC) and Nurick grades.

Results: TcMEP was recorded successfully in $0 \%, 28.6 \%, 72.3 \%$, and $100 \%$ of patients with MRC grades $1,2,3,4$, and 5 , respectively. SSEP was obtained from $0 \%, 37.5 \%, 21.5 \%, 61.4 \%$, and $85.4 \%$ of patients with MRC grades 1,2 , 3, 4, and 5 , respectively. TcMEP was recorded successfully in $84 \%$ of patients with Nurick grades $1-3$ and $26 \%$ of patients with Nurick grades 4-5. SSEPs were recorded successfully in $76.3 \%$ of patients with Nurick grades $1-3$ and $24 \%$ of patients with grades $4-5$. Conclusion: IONM during spine surgery may be useless in patients with MRC grades 1-2, applicable MRC grade 3, and useful MRC grades 4-5. MRC grade 3 is a critical point of indication for application of MEPs. In unmonitorable cases with MRC grade 3 , increasing stimulus intensity or facilitation techniques may be considered to improve the usefulness of TcMEP.

Key Words: Spine surgery $\cdot$ Intraoperative neuromonitoring $\cdot$ Motor weakness

\section{INTRODUCTION}

Intraoperative neuromonitoring (IONM) has been widely performed to prevent permanent neurological injury in highrisk spine surgeries. There is no definitely established consensus regarding the feasibility of transcranial motor evoked potential (TcMEP) monitoring, however TcMEP monitoring has been accepted as a useful method for early detection of neurological injury with high sensitivity ${ }^{6}$.

Generally, the reported success rate of basal MEPs recording was $79 \%-98 \%$ in patients without weakness ${ }^{2,89}$. However, application of IONM for patients with preoperative motor weakness is still controversial due to lower success rate and cost-effectiveness in spine surgery ${ }^{2,8}$. In addition, study regarding the success rate and feasibility of IONM in patients with

- Received: November 12, 2015 • Revised: February 3, 2016

- Accepted: February 5, 2016

Corresponding Author: In Ho Han, MD, PhD

Department of Neurosurgery, Pusan National University Hospital,

179 Gudeok-ro, Seo-gu, Busan 49241, Korea

Tel: +82-51-240-7257, Fax: +82-51-244-0282

E-mail: farlateral@hanmail.net

@This is an open access article distributed under the terms of the Creative Commons Attribution Non-Commercial License (http://creativecommons.org/licenses/by-nc/4.0/) which permits unrestricted non-commercial use, distribution, and reproduction in any medium, provided the original work is properly cited. preoperative motor weakness has rarely been reported and there are no definite guidelines for application of IONM to patients with motor weakness.

The purposes of our study are to evaluate the success rate and feasibility of IOM focusing on TcMEP for patients with preoperative motor weakness in spine surgery and suggest possible guidelines for application of IONM.

\section{MATERIALS AND METHODS}

\section{Patients Data}

Between November 2011 and December 2013, TcMEP and somatosensory evoked potential (SSEP) monitoring were attempted in 130 consecutive patients undergoing spine surgeries. Patients ranged in age from 14 to 81 years (mean standard deviation, $56.7 \pm 14.8$ years), and 84 patients were male. Inclusion criteria were cervical or thoracic cord lesions except for radiculopathy. Pathological conditions included 61 degenerative spinal diseases, 52 spinal tumors, 9 spinal traumas, and 2 vascular malformations. Preoperative motor function of the patients was classified according to the Medical Research Council (MRC) and Nurick grades (Table 1). The success rates of both SSEP and MEPs monitoring were assessed according to the preoperative MRC and Nurick grades. 
Table 1. The number of patients according to Medical Research Council (MRC) and Nurick grades

\begin{tabular}{lrrrccc}
\hline \hline & \multicolumn{7}{c}{ Grade } & Total \\
\cline { 2 - 6 } & 1 & 2 & 3 & 4 & 5 & \\
\hline MRC (n) & 2 & 8 & 14 & 65 & 41 & 130 \\
Nurick (n) & 46 & 15 & 36 & 12 & 21 & 130 \\
\hline
\end{tabular}

\section{TCMEP Monitoring Protocol}

TcMEPs were obtained using transcranial electrode stimulation, using the NIM-ECLIPSE monitoring system ver. 3.5.351 (Medtronic XOMED Inc., Memphis, TN, USA). Transcranial electrical stimulation was delivered by placing an anode $(2-\mathrm{cm}$ silver disc) at C3-4 (10-20 electrode system). A train of 6 pulses (each pulse 50-usec pulse width duration) with a 2-msec interval was used. To establish a baseline response, stimulus intensity was started at $200 \mathrm{~V}$ and gradually increased up to $400 \mathrm{~V}$. Muscle MEPs were recorded using needle electrodes in 4 extremities, including anterior tibialis muscles and anterior hallucis muscle. The time base was set at $100 \mathrm{msec}$ and the filter band pass was $100-5,000 \mathrm{~Hz}$. Any amplitude of muscle MEP in lower extremities was considered as a successful recording.

\section{SSEP Monitoring Protocol}

SSEP was monitored simultaneously with TcMEP in all cases. Briefly rectangular constant-current stimuli of 500-usec duration with intensities up to $30 \mathrm{~mA}$ were applied to either the median nerve at the wrist or the tibial nerve at the ankle at the stimulation rate of $3.9 \mathrm{~Hz}$. The upper extremity SSEP was recorded at $2 \mathrm{~cm}$ behind $\mathrm{C} 3$ and the lower extremity SSEP was recorded at $\mathrm{Cz}$ versus $\mathrm{Fz}$, with a bandpass from 30 to $3 \mathrm{kHz}$ and averaging 200 to 400 sweeps. Any amplitude of SSEP in P37 peak was considered as a successful recording. A 50\% drop in the SSEP amplitude or 10\% delay of SEP latency was defined as an abnormal finding.

\section{Anesthesia}

Anesthesia was induced with a constant infusion of thiopental or propofol (100 to $150 \mu \mathrm{g} / \mathrm{kg} / \mathrm{min})$, supplemented with remifentanil, with avoidance of bolus injections whenever possible. Vecuronium was administered to facilitate intubation and ventilation.

\section{RESULTS}

TcMEP was not obtained in any patients with MRC grades 1-2 (0\%). TcMEPs were obtained successfully in 4 out of 14 patients with MRC grade 3 (28.6\%), 47 out of 65 with MRC
Table 2. The successful rate of basal TcMEPs and SSEP recording according to MRC grade

\begin{tabular}{ccc}
\hline \hline MRC grade & Successful rate of TcMEP & Successful rate of SSEP \\
\hline 5 & $41 / 41(100)$ & $35 / 41(85.4)$ \\
4 & $47 / 65(72.3)$ & $40 / 65(61.5)$ \\
3 & $4 / 14(28.6)$ & $3 / 14(21.4)$ \\
2 & $0 / 8(0)$ & $3 / 8(37.5)$ \\
1 & $0 / 2(0)$ & $0 / 2(0)$ \\
\hline
\end{tabular}

Values are presented as number (\%).

TcMEP, transcranial motor evoked potential; SSEP, somatosensory evoked potential; MRC, Medical Research Council.

Table 3. The successful rate of basal TcMEPs and SSEP recording according to Nurick grade

\begin{tabular}{ccc}
\hline \hline Nurick grade & Successful rate of TcMEP & Successful rate of SSEP \\
\hline 1 & $46 / 46(100)$ & $40 / 46(87.0)$ \\
2 & $14 / 15(93.3)$ & $10 / 15(66.7)$ \\
3 & $24 / 36(66.7)$ & $24 / 36(66.7)$ \\
4 & $5 / 12(41.7)$ & $2 / 12(16.7)$ \\
5 & $4 / 21(19.0)$ & $2 / 21(9.5)$ \\
\hline
\end{tabular}

Values are presented as number (\%).

TcMEP, transcranial motor evoked potential; SSEP, somatosensory evoked potential.

grade 4 (72.3\%), and all patients with MRC grade $5(100 \%)$. SSEP was not obtained 2 patients with MRC grade $1(0 \%)$. SSEPs were obtained successfully in 3 out of 8 patients with MRC grade 2 (37.5\%), 3 out of 14 with grade 3 (21.4\%), 40 out of 65 with grade $4(61.5 \%)$, and 35 out of 41 with grade 5 (85.4\%) (Table 2).

TcMEP was recorded successfully in 84 out of 97 patients with Nurick grades 1-3 (86.6\%) and 9 out of 33 with Nurick grades 4-5 (27.3\%). SSEP was recorded successfully in 74 out of 97 patients with Nurick grades 1-3 (76.3\%) and 8 out of 33 with grades $4-5$ (24.2\%) (Table 3).

In 130 patients, there was no definite waveform loss of TcMEP in the lower extremity. In one patient undergoing cervical laminoplasty, a waveform of TcMEP disappeared in the right deltoid and triceps muscles and the wave form loss continued during surgery, but there was no noticeable motor weakness after surgery.

In one patient with MRC grade 3 in lower leg extremity who underwent intramedullary tumor surgery, a wave form of SSEP disappeared in lower extremity during surgery. Postoperatively, motor of lower extremity was aggravated from MRC grade 3 to 2 . In the patients, baseline TcMEP recording was initially not obtained.

\section{DISCUSSION}

Baseline TcMEP recording is affected by various factors, 
particularly anesthetic agent, muscle relaxants, and patient's vital status such as hypotension. These factors much influence on the stability of baseline TcMEP recording during surgery ${ }^{6,11)}$. However, these factors can be realized and corrected during surgery. Factors including age, lesion location, and preoperative neurological deficit can also influence the success rate of baseline TcMEP recording ${ }^{2}$. These factors are predetermined and important to determine the application of TcMEP due to a possible failure of baseline TcMEP recordings. The success rate of MEP recording is low in children younger than 7 years. In older aged groups, the success rate can decrease due to peripheral neuropathy or age-related disorders. Chen et $\mathrm{al}^{2)}$ reported higher success rates for lesions located in the cranial portion, compared with in spine lesions. In particular, preoperative motor weakness is significantly related to the success rate of baseline TcMEP recording because the nerve conduction pathway could already be dysfunctional. Several studies regarding the relation between preoperative motor deficit and the success rate of baseline MEP recording have been reported ${ }^{3,89}$. Chen et al..$^{2}$ reported that the success rate of lower extremity MEPs was lower at 39.1\% in patients with motor weakness compared with $78.9 \%$ in patients without motor weakness. They did not describe the exact grade of motor weakness. Rajshekhar et al. ${ }^{8)}$ reported a strong correlation between preoperative functional status and successful basal MEP recording. In the study, $91.4 \%$ of patients with MRC grade of 4 or 5 had a successful recording, but only $27.5 \%$ of patients with MRC grades $0-3$ had a successful recording.

In general, the success rate of TcMEP recording is low in patients with preoperative motor weakness. However, once the baseline TcMEP or SSEP is recorded successfully in patients with preoperative motor deficit, the feasibility of IONM in those patients can increase. Clark et al. ${ }^{3)}$ studied the usefulness of IONM with MEPs in patients with cervical and thoracic myelopathy. In the study, the sensitivity of MEPs was 100\% and positive prediction was $80 \%$ in patients with a preoperative motor deficit. Instead, the sensitivity of MEP was low at $50 \%$ in patients with normal motor.

In our result, $72.3 \%$ of patients with MRC grade 4 and $28.6 \%$ of patients with MRC 3 had a successful recording. All patients with normal motor had a successful baseline recording. On the contrary, the baseline TcMEP was not obtained in any patient with MRC grade 2 or less in the lower limbs. Therefore, the TcMEP recording may be useless in patients with MRC grade 2. Instead, the SSEP was recorded in $37.5 \%$ of patients with MRC grade 2. However, SSEP does not represent the real motor function of patients. Therefore, we suggest that the IONM including MEPs is useless in patients with MRC grade 2 or less in lower limbs, and the application of MEPs should be limited in those patients. In patients with MRC grade 3, TcMEP monitoring may still be controversial, considering its success rate ${ }^{7,8)}$. Similar to MRC grade, functional grade is also associated with the success rate of MEP and SSEP recordings. In our study, a good Nurick grade showed high successful recording of MEP and SSEP. Rajshekhar et al. ${ }^{8)}$ reported that functional grade is an independent predictor of a low success rate of MEPs recording. Nevertheless, TcMEP monitoring could be attempted in those patients because the methods to improve the success rate of baseline TcMEP recording have been reported ${ }^{1,4,5)}$. A technical modification of stimulation such as modifying the intensity of the stimulation current, duration, and interval between the stimulation is a basic method to improve the success rate of TcMEP monitoring. The most effective method to improve the success rate might be increasing the stimulus intensity, particularly in patients with preoperative motor weakness. However, complications of high voltage TcMEP stimulation include seizure, tongue bite, cardiac arrhythmias, and scalp burn ${ }^{10)}$. Therefore, increasing stimulus intensity is generally limited below maximal $1,000 \mathrm{~V}$.

Second, facilitating technique of TcMEP is another method to improve the success rate ${ }^{1,4,5}$. The concept of a facilitating technique is that a preconditioning pulse train preceding a multiple transcranial electrical stimulus leads to a larger MEP response. Journee et al. ${ }^{4)}$ reported that double-train stimulation can markedly facilitate a weak response from single-train stimulation and has been successful in patients with severe neuromuscular weakness, impaired spinal cord function, Duchenne muscular atrophy or Rett syndrome, and in a small number of neurologically normal patients. Although technical modification of stimulation may be useless in patients with MRC grade 2 or less, it can be adequately applied in patients with MRC 3. In addition, multimodality IONM including SSEP and electromyography could complement the insufficiency of TcMEP.

\section{CONCLUSION}

IONM during spine surgery may be useless in patients with MRC grades 1-2, applicable MRC grade 3, and useful MRC grades $4-5$. MRC grade 3 is a critical point of application for TcMEP. In unmonitorable cases with MRC grade 3, increasing stimulus intensity or facilitation techniques may be considered to improve the usefulness of TcMEP.

\section{CONFLICT OF INTEREST}

No potential conflict of interest relevant to this article was reported.

\section{ACKNOWLEDGMENTS}

This study was supported by clinical research grant (2015), Pusan National University Hospital.

\section{REFERENCES}

1. Andersson G, Ohlin A: Spatial facilitation of motor evoked res- 
ponses in monitoring during spinal surgery. Clin Neurophysiol 110:720-724, 1999

2. Chen X, Sterio D, Ming X, Para DD, Butusova M, Tong T, et al: Success rate of motor evoked potentials for intraoperative neurophysiologic monitoring: effects of age, lesion location, and preoperative neurologic deficits. J Clin Neurophysiol 24:281285, 2007.

3. Clark AJ, Ziewacz JE, Safaee M, Lau D, Lyon R, Chou D, et al: Intraoperative neuromonitoring with MEPs and prediction of postoperative neurological deficits in patients undergoing surgery for cervical and cervicothoracic myelopathy. Neurosurg Focus 35:E7, 2013

4. Journée HL, Polak HE, de Kleuver M, Langeloo DD, Postma AA: Improved neuromonitoring during spinal surgery using double-train transcranial electrical stimulation. Med Biol Eng Comput 42:110-113, 2004

5. Journée HL, Polak HE, De Kleuver M: Conditioning stimulation techniques for enhancement of transcranially elicited evoked motor responses. Neurophysiol Clin 37:423-430, 2007

6. Lall RR, Lall RR, Hauptman JS, Munoz C, Cybulski GR, Koski $\mathrm{T}$, et al: Intraoperative neurophysiological monitoring in spine surgery: indications, efficacy, and role of the preoperative check- list. Neurosurg Focus 33:E10, 2012

7. Nuwer MR, Emerson RG, Galloway G, Legatt AD, Lopez J, Minahan R, et al: Evidence-based guideline update: intraoperative spinal monitoring with somatosensory and transcranial electrical motor evoked potentials: report of the Therapeutics and Technology Assessment Subcommittee of the American Academy of Neurology and the American Clinical Neurophysiology Society. Neurology 78:585-589, 2012

8. Rajshekhar V, Velayutham P, Joseph M, Babu KS: Factors predicting the feasibility of monitoring lower-limb muscle motor evoked potentials in patients undergoing excision of spinal cord tumors. J Neurosurg Spine 14:748-753, 2011

9. Sala F, Palandri G, Basso E, Lanteri P, Deletis V, Faccioli F, et al: Motor evoked potential monitoring improves outcome after surgery for intramedullary spinal cord tumors: a historical control study. Neurosurgery 58:1129-1143, 2006

10. Schwartz DM, Sestokas AK, Dormans JP, Vaccaro AR, Hilibrand AS, Flynn JM, et al: Transcranial electric motor evoked potential monitoring during spine surgery: is it safe? Spine (Phila Pa 1976) 36:1046-1049, 2011

11. Stecker MM. A review of intraoperative monitoring for spinal surgery. Surg Neurol Int 3(Suppl 3):S174-187, 2012 